\title{
Hepatectomy combined with microwave ablation of the spleen for treatment of hepatocellular carcinoma complicated with splenomegaly: A retrospective study
}

\author{
JIAN-BO HAN ${ }^{1 *}$, FENG-WEI KONG ${ }^{2 *}$, HAI DING $^{1}$, YU-FENG ZHANG ${ }^{1}$, JUN-MAO LIU $^{1}$, \\ QIANG WEI ${ }^{3}$, LIANG HU ${ }^{1}$, LIANG ZHAO ${ }^{1}$, CHUAN-JUN XU ${ }^{4}$ and YONG-XIANG YI ${ }^{1}$ \\ ${ }^{1}$ Department of Hepatobiliary Surgery, The Second Hospital of Nanjing, Affiliated to Southeast University, \\ Nanjing, Jiangsu 210003; ${ }^{2}$ Department of General Surgery, Xuzhou Infectious Diseases Hospital, Xuzhou, Jiangsu 221004; \\ Departments of ${ }^{3}$ Interventional Ultrasound and ${ }^{4}$ Medical Imaging, The Second Hospital \\ of Nanjing, Affiliated to Southeast University, Nanjing, Jiangsu 210003, P.R. China
}

Received July 1, 2016; Accepted November 4, 2016

DOI: $10.3892 / \mathrm{mco} .2016 .1111$

\begin{abstract}
The present retrospective study aimed to investigate the mid-term safety and efficacy of hepatectomy combined with microwave ablation of the partial spleen for treatment of liver cancer complicated with hypersplenism. A retrospective analysis was performed on 23 patients who underwent hepatectomy combined with microwave ablation of the partial spleen for liver cancer, complicated with hypersplenism that was secondary to cirrhosis. The splenic and ablated volumes were calculated according to a contrast-enhanced computed tomography scan prior to and 2 weeks after the operation. Complete blood count and liver function tests were examined prior to and following the surgery, and complications and changes in the blood tests were monitored for 6 months. Over this period of investigation, the splenic volume was reduced by a mean value of $34.0 \%$. The levels of serum alanine aminotransferase and aspartate aminotransferase were increased on the first day after the operation $(\mathrm{P}<0.05)$, although they recovered to the normal level within 1 week $(\mathrm{P}<0.05)$. The total level of bilirubin increased slightly, along with moderately decreased levels of albumin and cholinesterase on the first day, although these changes were not significant compared with the baseline $(P>0.05)$. The white blood cell count was persistently significantly higher compared with the baseline
\end{abstract}

Correspondence to: Dr Yong-Xiang Yi, Department of Hepatobiliary Surgery, The Second Hospital of Nanjing, Affiliated to Southeast University, Zhongfu Road no. 1-1, Nanjing, Jiangsu 210003, P.R. China

E-mail: ian0126@126.com

${ }^{*}$ Contributed equally

Key words: microwave ablation, hypersplenism, hepatocellular carcinoma over the course of the 6 months $(\mathrm{P}>0.05)$. The platelet count did not increase significantly for the first week after the operation ( $\mathrm{P}>0.05)$; however, it was revealed to be significantly increased 1 month after the surgery $(\mathrm{P}<0.05)$. No significant complications were occurred during the follow-up period. In conclusion, hepatectomy combined with microwave ablation of the spleen was demonstrated to be a safe and effective procedure for patients with liver cancer and hypersplenism in the mid-term.

\section{Introduction}

Hepatocellular carcinoma (HCC) is a common disease in China, and patients with HCC typically suffer from liver cirrhosis and secondary hypersplenism. The blood platelet (PLT) and white blood cell (WBC) counts decrease dramatically, with abnormal coagulation function. The traditional therapy for hypersplenism is splenectomy, and the preferred treatment of HCC is hepatectomy, so the treatment advocated from previous studies is hepatectomy combined with splenectomy (1-4), although this procedure leads to severe coagulation disorder and lost immune function of the spleen; furthermore, the incidence of portal vein thrombosis is high following a splenectomy (5).

How to deal with hypersplenism and HCC simultaneously with reduced complications is an important issue. To reduce surgical trauma, Cheng et al (6) preferred local treatments for HCC, such as thermal ablation and transcatheter arterial chemoembolization, albeit with certain limitations. Partial splenic embolization in conjunction with percutaneous injection of ethanolamine oleate was also performed $(7,8)$. However, partial splenic embolization has not become a conventional treatment due to high complications, including splenic abscess, splenic rupture, gastrointestinal bleeding and a high mortality rate $(9-11)$.

Numerous studies have been concerned with thermal ablation for normal spleen, splenic injury and splenomegaly (12-14). Previous studies demonstrated that microwave ablation of the spleen for splenomegaly and hypersplenism had a favorable 
effect on the peripheral lymphocytes (15). This retrospective study aimed to evaluate the safety and efficacy of hepatectomy combined with microwave ablation of the spleen for HCC and hypersplenism.

\section{Patients and methods}

Patient enrollment. Between October 2011 and December 2012, 23 patients (16 men and 7 women) with HCC, splenomegaly and hypersplenism due to liver cirrhosis were treated with partial hepatectomy and microwave ablation of the spleen. The median age was 53 years. All the patients were infected with chronic hepatitis $\mathrm{B}$. The mean diameter of the liver tumors was determined to be $3.25 \pm 0.96 \mathrm{~cm}$; 11 of the tumors were located in the right posterior lobe, five were in the anterior lobe, four were in the left lateral lobe, two were in the left internal lobe, and one was in the caudate lobe. All patients presented with hepatic synthetic function as Child-Pugh class A or B.

Inclusion criteria. Inclusion criteria of patients in this retrospective study were as follows: WBC count $>4 \times 10^{9}$ cells $/ 1$ (normal level, $4-10 \times 10^{9}$ cells/1); blood PLT count $>100 \times 10^{9}$ cells $/ 1$ (normal level, $\sim 100-300 \times 10^{9}$ cells $/ 1$ ); and the presence of a solitary liver tumor with splenomegaly and hypersplenism secondary to liver cirrhosis, with liver function of Child-Pugh class A or B. The exclusion criteria were: Primary splenomegaly and hypersplenism; liver function of Child-Pugh class C; the presence of other immune system disorders, or severe cardiopulmonary diseases or portal vein/hepatic vein thrombosis; or patients with portal hypertension and esophageal and gastric varices.

Prior to the operation, laboratory data were obtained, including routine blood counts (the WBC and PLT counts), liver function, renal function, serum tumor markers $(\alpha$-fetoprotein, carcinoembryonic antigen, carbohydrate antigen 19-9) and a serum hepatitis B virus marker (hepatitis B surface antigen). In addition, all patients were examined using a contrast-enhanced computed tomography (CT) scanner (Light speed 32; Philips Healthcare, Andover, MA, USA). The splenic volume was calculated using three-dimensional (3D) contrast-enhanced CT. A Philips Brilliance CT workstation (Philip Medical Systems, Cleveland, OH, USA) was used for image post-processing. Microwave ablation of the spleen was performed prior to the removal of the liver tumor, and the splenic artery was ligated. Three points of puncture were selected in the upper, middle and lower areas of the spleen, respectively. Using an ultrasonic guide, the applicator shaft was punctured inside the spleen. The power of microwave ablation was set at $60 \mathrm{~W}$; the irradiation lasted for $10 \mathrm{~min}$ in every point of puncture. Subsequently, resection of the liver tumor was performed.

Statistical analysis. All statistical analyses were performed using SPSS software, version 17.0 (SPSS, Inc., Chicago, IL, USA). Changes in the WBC and the PLT counts and liver function prior to and following the operation were evaluated using the paired Student's $t$-test. $\mathrm{P}<0.05$ was considered to indicate a statistically significant difference.

\section{Results}

The CT scan revealed hypoattenuating ablation areas in the spleen following the operation. The contrast-enhanced CT scan revealed no enhancement in the ablation area, suggesting complete necrosis of the ablated splenic parenchyma (Fig. 1). The surface of the spleen was no longer smooth, as indicated by the $3 \mathrm{D}$ contrast-enhanced CT scans (Fig. 2). The average ablation percentage was determined to be $34.20 \pm 1.72 \%$.

No severe complications or surgery-associated mortality occurred. The predominant adverse effect noted was fever, which was present in 2 of the 23 patients, with a highest temperature recorded of $38.7^{\circ} \mathrm{C}$. Spleen hematoma occurred in one patient, although it was absorbed gradually within a period of 3 months (Fig. 3). Only one patient developed an abdominal hemorrhage $8 \mathrm{~h}$ after the operation and, consequently, a second operation was performed for hemostasis.

The mean WBC count prior to the operation was $(3.54 \pm 1.04) \times 10^{9}$ cells/1, which peaked at one day $[(11.58 \pm 4.89)$ $\mathrm{x} 10^{9}$ cells/1] after the procedure, and subsequently gradually decreased over time, until a stable level was maintained $\left(>5.0 \times 10^{9}\right.$ cells/l) one month later. The WBC count still remained normal $\left(>4.0 \times 10^{9}\right.$ cells $\left./ 1\right) 6$ months after the operation (Fig. 4A). The mean PLT count prior to hepatectomy and microwave ablation was $(42.57 \pm 18.59) \times 10^{9}$ cells $/ 1$, and the count began to increase gradually after the treatment, reaching a peak level at 14 days after the operation, with a mean peak PLT count of $(105.43 \pm 46.28) \times 10^{9}$ cells/l; subsequently it was decreased over time. The PLT count was significantly different after the treatment compared with that prior to surgery $(\mathrm{P}<0.05)$ (Fig. 4B). Statistical analysis revealed a persistent significant increase in the WBC count between 1 day and 6 months after the treatment $(\mathrm{P}<0.05)$ (Fig. 4).

The levels of serum alanine aminotransferase and aspartate aminotransferase increased significantly during the first day following the hepatectomy and microwave ablation of the spleen $(\mathrm{P}<0.05)$, although they fell back to normal levels within one week. The total bilirubin level increased steadily, whereas the level of albumin decreased slightly, without any significant changes being observed compared with those levels observed prior to the operation (Fig. 5).

\section{Discussion}

The patients involved in the present study suffered from HCC and hypersplenism, which had to be resolved simultaneously for the following reasons. First, the sharp decrease in the levels of WBCs and PLTs caused by hypersplenism raises the incidence of infection and hemorrhage, and secondly, chemotherapy or radiation therapy occasionally may be the only treatment for tumor recurrence following liver resection, although, since the counts of WBCs and PLTs are lower, these treatments would consequently be delayed. Hypersplenism and splenomegaly for liver cancer patients may be treated with minimally invasive surgery, such as partial splenic embolization, splenic irradiation and percutaneous injection of ethanolamine oleate, although these treatments are often associated with limitations and high complications (16-20).

Wood and Bates (21) first reported radiofrequency ablation of the spleen for splenic metastasis in 2001. Liu et al (22) 

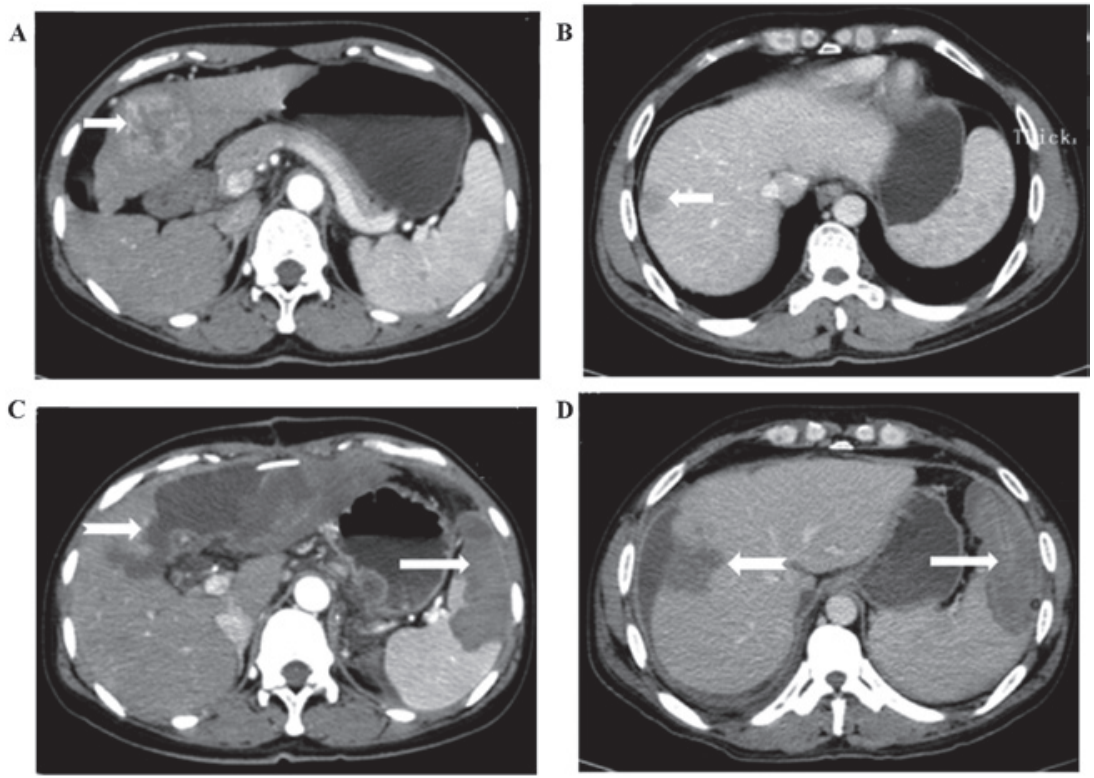

Figure 1. Representative CT scans of one patient with hepatocellular carcinoma and hypersplenism due to liver cirrhosis who underwent partial hepatectomy and microwave ablation of the spleen. (A and B) Contrast-enhanced CT scans, revealing one neoplasm (indicated by the short arrows) in the left liver lobe and splenomegaly according to the diagnostic criteria. (C and D) Contrast-enhanced CT scans of the transverse section, revealing an unenhanced low-attenuation microwave ablation lesion (indicated by the long arrow) in the spleen, and an unenhanced low-density area, termed the remaining surgery cavity, after partial hepatectomy (shown by the dovetail arrow) in the left lobe of the liver 2 weeks after the operation. CT, computed tomography.

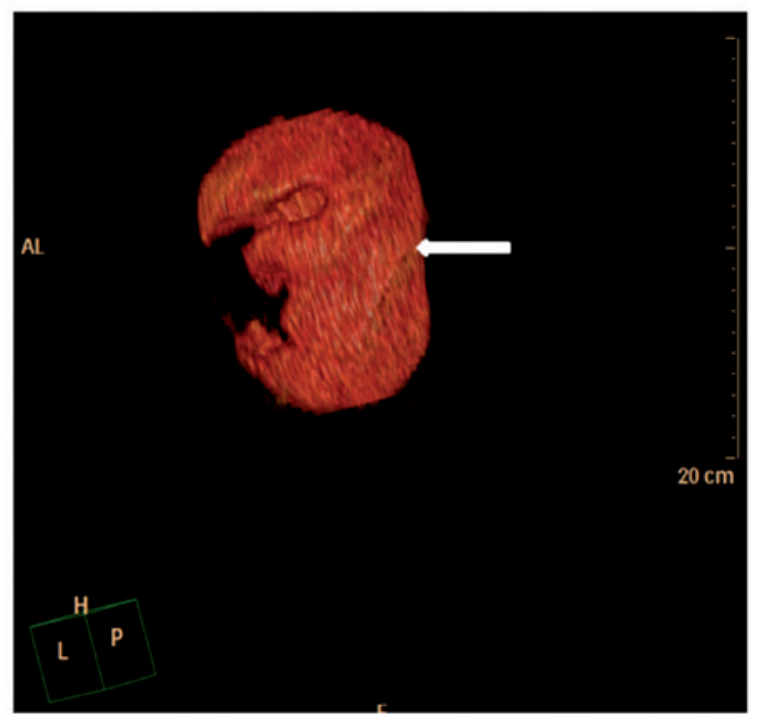

Figure 2. Calculation of the splenic ablated volume. The splenic ablated volume was calculated on three-dimensional contrast-enhanced computed tomography scans 2 weeks after the operation. The surface of the spleen was no longer smooth, and the depressed areas were ablated lesions. One of the ablated areas (indicated by the long arrow) is highlighted.

concluded that radiofrequency ablation of the spleen was a safe and effective procedure. The mechanism of radiofrequency ablation was the same as microwave ablation. The spleen vasculature was occluded by venous microthrombi as the spleen was ablated, and subsequently, the infarction focus caused by the vascular thrombosis was steadily absorbed. Microwave ablation of the spleen combined with partial hepatectomy may offer one possible treatment for HCC and hypersplenism, although the safety and mid-term effects of this procedure have yet to be fully elucidated.
Microwave ablation of the spleen exerted a positive influence on liver function (23-27). The pressure of the portal vein decreased after microwave ablation of the spleen, which helped to relieve the hepatic parenchymal congestion and hepatocyte swelling $(28,29)$. The blood flow was reduced in the portal vein, and subsequently increased in the hepatic artery, and therefore liver regeneration became more active due to enhanced oxygen exchange of the hepatic cells with markedly improved liver function (30). The liver cells were regenerated more effectively following microwave ablation of the spleen. In the present study, the liver function recovered within one week following the operation after simultaneous hepatectomy and microwave ablation of the spleen. A reduced portal vein blood flow led to a decrease of pressure in the portal vein, which helped to reduce the risk of bleeding to the esophageal and gastric varices.

The spleen artery was ligated to prevent blood oozing out when the needle was punctured and pulled out, and heat energy generated by the microwave ablation itself was also able to prevent bleeding (31). The findings of the present study indicated that the amount of blood PLTs was significantly higher 2 weeks after the surgery $(\mathrm{P}<0.05)$, although the difference between the preoperative number of blood PLTs and those present one week later was not significant $(\mathrm{P}>0.05)$. The number of PLTs clearly increased, which led to the formation of portal vein thrombosis following splenectomy, with the rate of thrombosis up to $34.2 \%$ (32). Several of the patients required anticoagulant therapy. After microwave ablation of the partial spleen, the PLT count began to increase steadily, reaching a peak at 14 days after the operation, and subsequently, the count decreased over time. The levels were maintained to the extent that preventable anticoagulant therapy was not required, and no case 

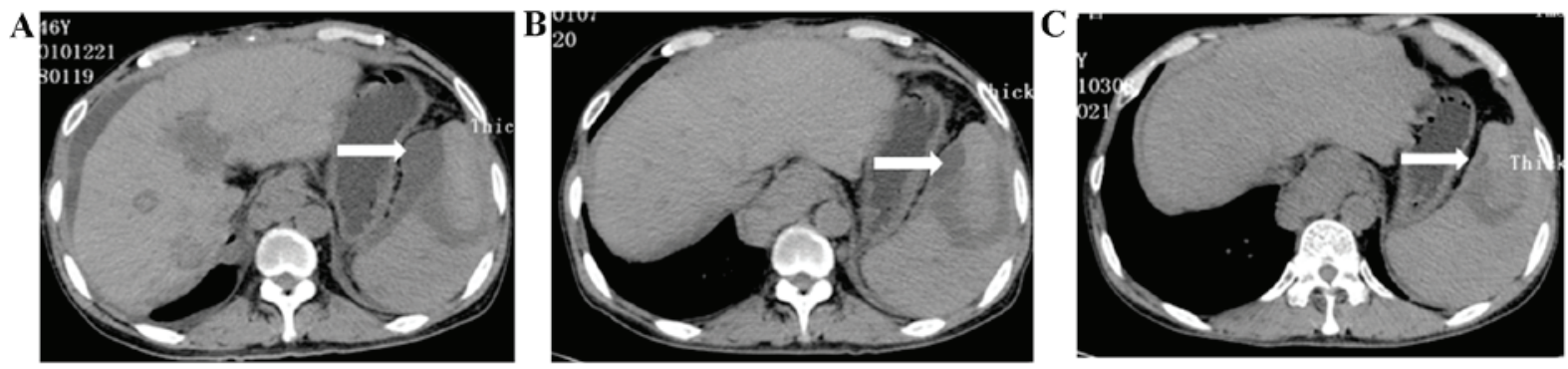

Figure 3. Spleen hematoma occurred in one patient following microwave ablation of the spleen. The plain CT scan revealed a lower-density area (indicated by the long arrow) under the spleen membrane, and the area began to shrink: As revealed on the routine plain CT scan following the operation, the spleen hematoma was absorbed gradually. (A) A plain CT scan, revealing the spleen hematoma 2 weeks after the operation. (B) The spleen hematoma had decreased in size one month later. (C) Spleen hematoma was absorbed almost completely 3 months post-operation.
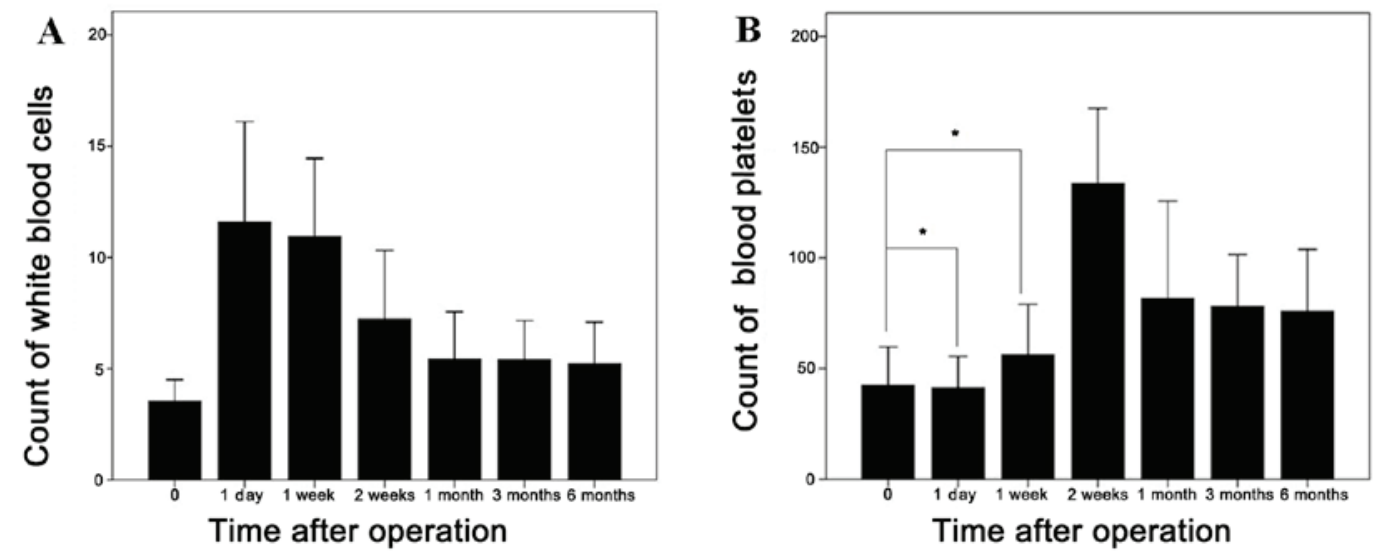

Figure 4. Changes in the counts of white blood cell and blood platelets after the operation. (A) The white blood cell count peaked on day 1 after the procedure, and subsequently gradually decreased over time, arriving at a stable level one month later. The white blood cell count still remained at the normal level 6 months after the operation. (B) The blood platelet count began to increase gradually after the treatment, and reached a peak level at 2 weeks after the operation; however, the platelet count did not increase significantly during the first week after the operation. "P $>0.05$ vs. the pre-operation.

with portal vein thrombosis was detected over the course of the next 6 months.

The areas of the spleen that were chosen for ablation were a constant source of debate. The volume of the necrotic area of the spleen was the crucial aspect that determined the surgical effect. In the present study, three points of puncture in the upper, middle and lower areas of the spleen, respectively, were selected. The adjacent organs were isolated from the spleen with wet saline gauze prior to microwave ablation of the spleen. Injuries to the surrounding tissues, including the stomach, colon, diaphragm, kidney and pancreatic tail, were not observed, and only three cases had complications, where left pleural effusion was observed, which was diminished slightly without treatment.

In conclusion, microwave ablation of the spleen combined with partial hepatectomy is a safe and effective technique for treatment of HCC and hypersplenism, and the PLT and WBC counts were increased significantly, at the same time, with fewer complications.

\section{Acknowledgements}

This study was supported by the Nanjing Medical Science and Technique Development Foundation, Nanjing Department of Health (grant nos. QRX11235 and ZDX12008) and the

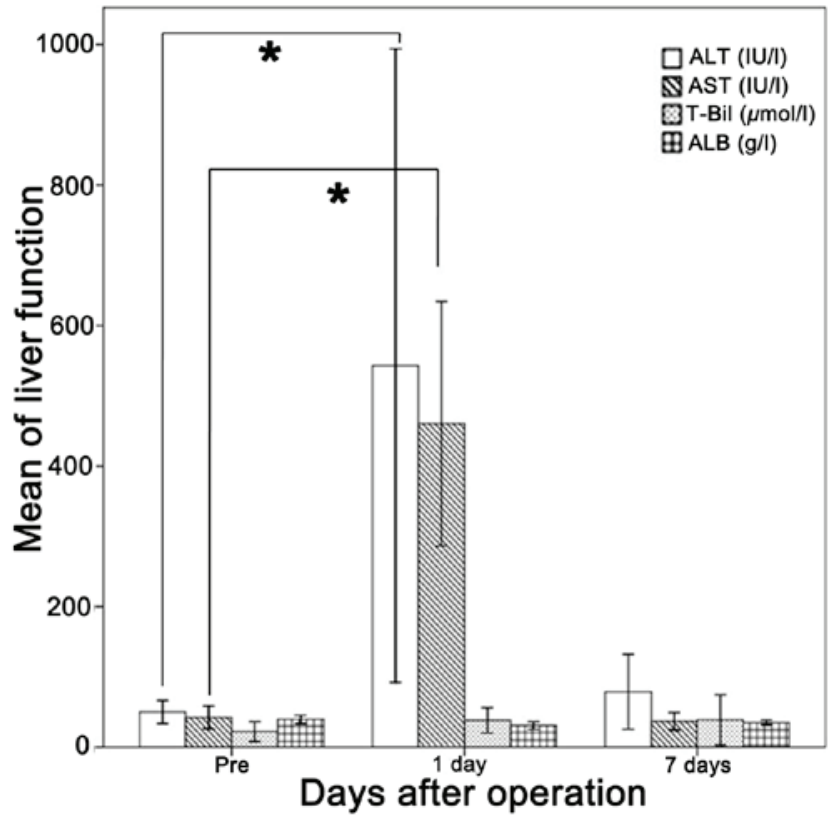

Figure 5. Changes in liver function following the operation. Levels of ALT and AST increased significantly on the first day after the operation, although they declined to the normal or preoperative level within one week. T-Bil increased steadily, whereas the levels of albumin deceased slightly. ${ }^{*} \mathrm{P}<0.05$ vs. pre-operation. ALT, alanine aminotransferase; AST, aspartate aminotransferase; T-Bil, total bilirubin; ALB, albumin. 
Jiangsu Science and Technology Project of Clinical Medicine Foundation, Science and Technology Department of Jiangsu Province (grant no. BL2014005).

\section{References}

1. Nanashima A, Abo T, Takeshita H, Yamasaki N, Sawai T and Nagayasu T: Safety of hepatectomy accompanying combined resection of other organs. Hepatogastroenterology 59: 1915-1920, 2012.

2. Wang C, Li C, Wen TF, Yan LN, Li B, Liang GL and Li KW: Safety of synchronous hepatectomy and splenectomy for patients with hepatocellular carcinoma and hypersplenism Hepatogastroenterology 59: 526-528, 2012.

3. Ohno T, Furui J, Hashimoto T, Hirabaru M, Kohno M, Iwao A and Koyamatsu J: Simultaneous laparoscopic hand-assisted hepatectomy and splenectomy for liver cancer with hypersplenism: Report of a case. Surg Today 41: 444-447, 2011.

4. Aiyama T, Kamiyama T, Nakanishi K, Yokoo H, Taniguchi M, Fukumori D, Tahara M, Kakisaka T, Kamachi H, Matsushita M and Todo S: A study of cirrhotic patients with hepatocellular carcinoma and huge splenomegaly who received treatment for hepatocellular carcinoma with concomitant splenectomy. Gan To Kagaku Ryoho 37: 2683-2686, 2010 (In Japanese).

5. Ushitora Y, Tashiro H, Takahashi S, Amano H, Oshita A, Kobayashi T, Chayama K and Ohdan H: Splenectomy in chronic hepatic disorders: Portal vein thrombosis and improvement of liver function. Dig Surg 28: 9-14, 2011.

6. Cheng JW and Lv Y: New progress of non-surgical treatments for hepatocellular carcinoma. Med Oncol 30: 381, 2013.

7. Koconis KG, Singh H and Soares G: Partial splenic embolization in the treatment of patients with portal hypertension: A review of the English language literature. J Vasc Interv Radiol 18: 463-481, 2007.

8. Shina S, Aoyama H, Shiratori Y, Mutoh H, Kurita M, Ota S, Terano A and Sugimoto T: Ultrasound-guided percutaneous injection of ethanolamine oleate for hypersplenism. An experimental study in dogs. Invest Radiol 25: 651-657, 1990.

9. Matsumoto T, Yamagami T, Terayama K, Kato T, Hirota T, Yoshimatsu R, Miura H, Ito H, Okanoue T and Nishimura T: Risk factors and clinical course of portal and/or splenic vein thrombosis after partial splenic embolization. Acta Radiol 50: 617-623, 2009

10. Hayashi H, Beppu T, Okabe K, Masuda T, Okabe H and Baba H: Risk factors for complications after partial splenic embolization for liver cirrhosis. Br J Surg 95: 744-50, 2008.

11. Wang HY, Shih SC, Lin SC, Chang WS, Wang TE, Lin FJ and Yang FS: Partial splenic embolization: 12-month hematological effects and complications. Hepatogastroenterology 55: $1838-1842,2008$.

12. Li Y, Cui L, Zhang W, Tian Y and Li M: Laparoscopic radiofrequency ablation for traumatic splenic rupture. J Surg Res 185: 711-716, 2013.

13. Feng K, Ma K, Liu Q, Wu Q, Dong J and Bie P: Randomized clinical trial of splenic radiofrequency ablation versus splenectomy for severe hypersplenism. Br J Surg 98: 354-361, 2011.

14. Liu Q, Ma K, He Z, Dong J, Hua X, Huang X and Qiao L: Radiofrequency ablation for hypersplenism in patients with liver cirrhosis: A pilot study. J Gastrointest Surg 9: 648-657, 2005.

15. Liang P, Gao Y, Zhang H, Yu X, Wang Y, Duan Y and Shi W: Microwave ablation in the spleen for treatment of secondary hypersplenism: A preliminary study. AJR Am J Roentgenol 196: 692-696, 2011.

16. Ishikawa $\mathrm{T}$, Kubota $\mathrm{T}$, Horigome R, Kimura N, Honda H, Iwanaga A, Seki K, Honma T and Yoshida T: Concurrent partial splenic embolization with transcatheter arterial chemoembolization for hepatocellular carcinoma can maintain hepatic functional reserve. Hepatol Res 44: 1056-1061, 2014.
17. Hidaka H, Kokubu S, Nakazawa T, Minamino T, Takada J, Tanaka Y, Okuwaki Y, Watanabe M, Shibuya A and Saigenji K: Therapeutic benefits of partial splenic embolization for thrombocytopenia in hepatocellular carcinoma patients treated with radiofrequency ablation. Hepatol Res 39: 772-778, 2009.

18. Harao M, Beppu T, Masuda T, Hayashi H, Okabe H, Okabe K, Imseung $\mathrm{C}$, Komori $\mathrm{H}$, Horino $\mathrm{K}$ and Baba $\mathrm{H}$ : The significance of combined treatment for hepatocellular carcinoma with partial splenic embolization and transcatheter arterial chemoembolization using IA call/lipiodol. Gan To Kagaku Ryoho 35: 2027-2079 2008 (In Japanese).

19. Huang JH, Gao F, Gu YK, Li WQ and Lu LW: Combined treatment of hepatocellular carcinoma with partial splenic embolization and transcatheter hepatic arterial chemoembolization. World J Gastroenterol 13: 6593-6597, 2007.

20. Shimizu T, Tajiri T, Yoshida H, Mamada Y, Taniai N, Kawano Y, Takahashi T, Arima Y, Aramaki T and Kumazaki T: Hand-assisted laparoscopic hepatectomy after partial splenic embolization. Surg Endosc 17: 1676, 2003.

21. Wood BJ and Bates S: Radiofrequency thermal ablation of a splenic metastasis. J Vasc Interv Radiol 12: 261-263, 2001.

22. Liu QD, Ma KS, He ZP, Ding J, Hung XQ and Dong JH: Experimental study on the feasibility and safety of radiofrequency ablation for secondary splenomagely and hypersplenism. World J Gastroenterol 9: 813-817, 2003.

23. Nasr MM, Hassan AM, Elsebaie SB, Elsebae MA and Nosseir MM: Effect of splenectomy on liver regeneration and function following partial hepatectomy: Experimental study. J Egypt Soc Parasitol 41: 601-610, 2011.

24. Arakawa Y, Shimada M, Utsunomya T, Imura S, Morine Y, Ikemoto $\mathrm{T}$ and Takasu C: Effects of splenectomy on hepatic gene expression profiles after massive hepatectomy in rats. J Gastroenterol Hepatol 28: 1669-1677, 2013.

25. Kim J, Kim CJ, Ko IG, Joo SH and Ahn HJ: Splenectomy affects the balance between hepatic growth factor and transforming growth factor- $\beta$ and its effect on liver regeneration is dependent on the amount of liver resection in rats. J Korean Surg Soc 82: 238-235, 2012

26. Ren YS, Qian NS, Tang Y, Liao YH, Liu WH, Raut V, Tao KS and Dou KF: Beneficial effects of splenectomy on liver regeneration in a rat model of massive hepatectomy. Hepatobiliary Pancreat Dis Int 11: 60-65, 2012.

27. Arakawa Y, Shimada M, Uchiyama H, Ikegami T, Yoshizumi T, Imura S, Morine $\mathrm{Y}$ and Kanemura $\mathrm{H}$ : Beneficial effects of splenectomy on massive hepatectomy model in rats. Hepatol Res 39: 391-397, 2009.

28. Zipprich A, Steudel N, Behrmann C, Meiss F, Sziegoleit U, Fleig WE and Kleber G: Functional significance of hepatic arterial flow reserve in patients with cirrhosis. Hepatology 37 : 385-392, 2003.

29. Eipel C, Abshagen K, Ritter J, Cantré D, Menger MD and Vollmar B: Splenectomy improves survival by increasing arterial blood supply in a rat model of reduced-size liver. Transpl Int 23: 998-1007, 2010.

30. Liu QD, Ma K, Song Y, Zhou N and He Z: Two-year follow-up of splenic radiofrequency ablation in patients with cirrhosis hypersplenism: Dose increased hepatic arterial flow induce liver regeneration? Surgery 143: 509-518, 2008.

31. Goldberg SN, Solbiati L, Halpern EF and Gazelle GS: Variables affecting proper system grounding for radiofrequency ablation in an animal model. J Vasc Interv Radiol 11: 1069-1075, 2000.

32. Ushitora Y, Tashiro H, Takahashi S, Amano H, Oshita A, Kobayashi T, Chayama K and Ohdan H: Splenectomy in chronic hepatic disorders: Portal vein thrombosis and improvement of liver function. Dig Surg 28: 9-14, 2011. 